\title{
Challenge is the key to stroke rehabilitation.
}

\author{
Faizan Kashoo*, Mehrunnisha Ahmad \\ Majmaah University, College of Applied Medical Sciences, Majmaah, Riyadh, Saudi Arabia
}

\begin{abstract}
Trunk balance is a complex interplay of precise neuromuscular coordination, and such interaction requires the active involvement of voluntary postural control, anticipatory postural reactions, and reactive postural control. Stroke affects trunk balance, resulting in a delay in the acquisition of essential functional skills, and affects postural symmetry and the ability to shift weight as per the demand of a task. Trunk balance training involves various advanced methods, such as robotic training, video gaming, and force platforms, but they provide insufficient evidence to support the training and reinforcement of weak postural reactions. The primary aim of this letter is to emphasize the importance of trunk training, which involves unpredictable multi-planar tilting of the sitting support surface, to reinforce the postural reactions of trunk balance.
\end{abstract}

Keywords: Trunk balance, Postural symmetry.

Accepted on November 1, 2018

\section{Introduction}

The functional limitations experienced after a stroke vary from individual to individual, mandating a customized rehabilitation approach [1]. Poor sitting ability is a frequent impairment experienced after stroke. The improvement of trunk balance is important for individuals because sitting is a vital skill for independent living. Furthermore, sitting ability is a useful prognostic indicator of outcomes after stroke [2]. A large amount of research has stated that the loss of muscle dexterity, weakness, and weak postural reactions result in poor trunk balance [3]. Trunk reactions, such as postural, equilibrium and anticipatory postural reactions, are integral parts of sitting balance $[4,5]$. The research literature contains insufficient evidence on techniques used by clinicians to facilitate these reactions. In a normal individual during functional activity, the anticipatory postural reactions make necessary adjustments of body segments, providing a stable foundation for an intended functional activity. Equilibrium and postural reactions make additional segmental adjustments, thereby retaining the centre of gravity within the base of support and increasing the range of functional activity. Trunk balance retraining programmes include activities such as reaches beyond arm length, multidirectional nudges, use of the Bon Saint Côme device for axial postural rehabilitation and force-platform feedback training, but none of these approaches have proven effective [6]. Trunk training devices utilize two types of support surfaces: static and dynamic. Patients using the video game technique (dynamic support surface) learn tricks to reach to their goal without challenging the limits of their trunk stability. Dynamic support surfaces used by video gaming rehabilitation are controlled by patients. These patients tend to place their activity at minimally challenging levels or, in some instances, restrict postural adjustment to a single plane (mono-planar segmental adjustment). Therefore, we need to develop a trunk balance training device that creates a challenging situation by providing multi-planar unpredictable tilts of varying speed, direction and degree of a support surface. The movement of the support surface will have six degrees of freedom: 3 linear and 3 rotational movements along the $\mathrm{x}, \mathrm{y}$ and $\mathrm{z}$ cardinal planes and axes. Several combinations of movements coupled with varying speeds, directions and angles will create a challenging environment to facilitate trunk reactions.

Training that involves unpredictable tilts in various planes of movement may facilitate postural reactions better than relying on stable and unstable bases of support alone. In an ideal clinical setting, such training would comprise a rocker board placed on a high table. Patients seated on the high table will perform various functional activities with the base of support tilted in various directions. We observed in our clinical setting that a small amount of controlled tilting of the support surface will result in a large degree of segmental adjustment and strong facilitation of trunk reactions. This system of retraining trunk balance can become precise and accurate by adding computerized controlled multi-directional tilts, coupled with pressure sensors on the seating surface, providing visual biofeedback on symmetrical weight bearing.

\section{Conclusion}

Unpredictable tilting of the support surface in sitting, along with the performance of functional activities, would prove challenging to the patient. This system of retaining sitting balance warrants future research involving comparisons between the facilitation of postural reactions through perturbation from the upper and lower trunk, the angle of the 
tilt, the direction of the tilt and the speed of the tilt of the support surface.

\section{References}

1. Hugues A, Di Marco J, Janiaud P, Xue Y, Pires J, Khademi H. Efficiency of physical therapy on postural imbalance after stroke: study protocol for a systematic review and meta-analysis. Vol. 7, BMJ Open. London BMJ Publishing Group LTD 2017; e013348.

2. Sandin KJ, Smith BS. The measure of balance in sitting in stroke rehabilitation prognosis. Stroke J Cerebral Circul USA 1990; 21: 82-86.

3. Karthikbabu S, Chakrapani M, Ganesan S, Ellajosyula R. Relationship between pelvic alignment and weightbearing asymmetry in community-dwelling chronic stroke survivors. J Neurosci Rural Pract Mumbai Medknow Publ Media Pvt. Ltd. 2016; 7; 37-40.

4. Kashoo F, Baradie R. Clinical trial results-rehabilitation and recovery comparing the immediate effect of auditory and visual mental imagery on reaching and grasping task among sub-acute stroke patients: esoc-0896. Int J Stroke 2015; 10: 89-90.

5. Kashoo F, Baradie R. Motorized multidirectional protuberance device for sitting balance of stroke patients. Int J Stroke 2015; 89.

6. Geurts ACH, de Haart M, van Nes IJW, Duysens J. A review of standing balance recovery from stroke. Gait Posture Netherlands Elsevier 2005; 22: 267-281.

\section{*Correspondence to}

Faizan Kashoo

Majmaah University

College of Applied Medical Sciences

Saudi Arabia 